\title{
La Influencia del Aprendizaje Significativo de Ausubel en el Desarrollo de las Técnicas de Escritura Creativa de Rodari
}

\author{
The Influence of Ausubel's Significant Learning on the Development of Rodari's \\ Creative Writing Techniques
}

Johanna Marcela Rozo

\section{○ EDICIÓN: 进e-CIVTAC}

Recibido: 11/julio/2020

Aceptado: 9/agosto/2020

Publicado: 25/septiembre/2020

${ }^{1}$ Colombia

\section{IIIIInstitución}

${ }^{1}$ Universidad de Pamplona

\section{Correo Eletrónico}

1johanna.rozo@unipamplona.edu.co

(D) ORCID

1https://orcid.org/0000-0003-3842-6931

\section{Citar así: GCAPA / IEEE}

Rozo, J. (2020). La Influencia del Aprendizaje Significativo de Ausubel en el Desarrollo de las Técnicas de Escritura Creativa de Rodari . Revista TecnológicaEducativa Docentes 2.0, 9(2), 88-94. https://doi.org/10.37843/rted.v9i2.149

\footnotetext{
J. Rozo, "La Influencia del Aprendizaje Significativo de Ausubel en el Desarrollo de las Técnicas de Escritura Creativa de Rodari ", RTED, vol. 9, n. ${ }^{\circ}$ 2, pp. 88-94, sep. 2020.
}

\section{Resumen}

La investigación se basó en el estudio del aprendizaje significativo Ausubel, aplicando las técnicas de escritura de Rodari. Los términos producción literaria, y proceso creativo, no son muy conocidos en los ambientes escolares. La investigación tuvo como objetivo el análisis de la influencia del aprendizaje significativo de Ausubel (1963) en el desarrollo de las técnicas de escritura de Rodari (1999) con los estudiantes de primer semestre de Comunicación Social - Universidad de Pamplona. Para tal efecto se realizó una revisión bibliográfica sobre los aspectos relevantes de la teoría estudiada, así como de los trabajos en el ámbito internacional, nacional y regional, los cuales fueron de gran utilidad para el diseño metodológico, el planteamiento de la estrategia y el análisis de los resultados. El tipo de investigación fue cualitativo con el método fenomenológico, esta investigación inicia con una etapa de diagnóstico donde se logró establecer una población de estudio tenía conceptos errados sobre creación literaria, prejuicios a la hora de escribir que les impedían generar un proceso creativo. Luego de aplicar las técnicas de recolección de información, de hacer el análisis de las categorías se llegó a la conclusión que la implementación de estrategias pedagógicas del aprendizaje significativo en las técnicas de creación de G. Rodari permite construcción de conocimientos y desarrollo de habilidades de escritura literaria en el estudiante donde este desarrolla un aprendizaje en construcción de textos a través del manejo de técnicas que potencian la creatividad.

Palabras clave: Aprendizaje significativo, técnicas de escritura, escritura creativa, producción literaria, proceso creativo.

\begin{abstract}
The research was based on the study of Ausubel meaningful learning, applying Rodari's writing techniques. The terms literary production, and creative process, are not well known in school environments. The objective of the research was to analyze the influence of Ausubel's (1963) significant learning in the development of Rodari's (1999) writing techniques with first-semester students of Social Communication - University of Pamplona. For this purpose, a bibliographic review was carried out on the relevant aspects of the theory studied, as well as the works in the international, national and regional fields, which were very useful for the methodological design, the approach of the strategy and the analysis. From the results. The type of research was qualitative with the phenomenological method, this research begins with a diagnostic stage where it was possible to establish a study population had misconceptions about literary creation, prejudices at the time of writing that prevented them from generating a creative process. After applying the information gathering techniques, after doing the analysis of the categories, it was concluded that the implementation of pedagogical strategies for meaningful learning in G. Rodari's creation techniques allows the construction of knowledge and the development of writing skills. literary in the student where he develops learning in the construction of texts through the management of techniques that enhance creativity.
\end{abstract}

Keywords: Meaningful learning, writing techniques, creative writing, literary production, creative process. 


\section{Introducción}

La investigación se basó en el estudio del aprendizaje significativo Ausubel, aplicando las técnicas de escritura de Rodari. Los términos producción literaria, y proceso creativo, no son muy conocidos en los ambientes escolares. Los docentes de español, aunque sean muy doctos en su área desconocen, queriendo o sin querer, los procesos de estimulación de la creatividad literaria. En las escuelas todavía se escuchan las palabras "escriban un cuento" una orden que se da sin más instrucción que "inspírense". Acto seguido encontramos estudiantes que no escriben, que escriben muy poco y el resultado son trabajos sin estructura narrativa, no son cuentos ni son relatos. Son apenas un esbozo de historias sin mayor profundidad.

Esto se repite a lo largo del proceso educativo y cuando los estudiantes llegan a la universidad como en el caso de los estudiantes de primer semestre de Comunicación Social desconocen el proceso de escritura y a muchos les parece difícil, tedioso, complicado y a estas alturas de su formación escolar se han escudado en la palabra "inspiración", palabra muy utilizada y que ha perdido su sentido, ya que por su facilismo impide que los estudiantes vean en la creación literaria (cuentos, poemas, relatos incluso novelas) como un oficio que implica aprender conceptos, desarrollar habilidades y generar un proceso de aprendizaje con apropiación de conocimiento.

En este sentido, la teoría de Ausubel resultó apropiada para generar un proceso educativo que debe ser lúdico, donde se deben tener en cuenta los procesos y la apropiación de esos conocimientos para el desarrollo de actividades lecto-escritoras. Las técnicas creadas y aplicadas por Rodari, técnicas que se retomaran para generar un proceso de producción escrita desde la literatura. Uno de los términos que aporta Rodari es la fantástica como complemento de la lógica, esta última tan privilegiada en las aulas de clase.

Para este trabajo se incluyeron investigaciones que a nivel regional, nacional e internacional han aportado al tema de la construcción de aprendizaje en la creación literaria con diferentes técnicas, por otra parte, se entrevistó a tres directores de los talleres de escritura creativa Relata para que desde su experiencia le aportaran conocimiento a esta investigación.

Luego de este trabajo investigativo, de aplicar las las técnicas de recolección de información que desde el método fenomenológico nos proporciona, se realizó el análisis de la información a partir de las categorías propuestas que llevaron finalmente a los resultados, conclusiones, recomendaciones, que dan cuenta de lo apropiado de la teoría de Ausubel y de la aplicación de técnicas de escritura de Rodari.

\section{Metodología}

\section{Preliminares}

Los estudiantes de Comunicación Social de la Universidad de Pamplona tienen la intención de escribir literatura. Pero, el problema se enfoca en que no saben cómo hacerlo por que parten de muchos prejuicios que se fueron creando a través de su proceso educativo $y$, por otra parte, tienen una visión de la escritura literaria errada. Domínguez (2002) en su estudio ¿Por qué no escriben textos los estudiantes? Explica:

\begin{abstract}
En el país la comunicación escrita no es parte de los aprendizajes que proporciona la educación formal. En general, el mexicano alfabetizado puede leer y escribir (deletrear y gabaratear), manejar números, expresar cantidades simbólicamente y comunicarse, pero no consigue (o no sabe cómo) manifestar sus pensamientos, expresar correcta o adecuadamente sus conocimientos por escrito en los diversos géneros textuales universitarios. (p. 3).
\end{abstract}

Lo anterior es similar en muchas universidades de Colombia. La ven como un "don divino" se alejan de los puntos importantes de la escritura que es primero la disciplina y el oficio; por otra parte, la investigación que ayuda a construir la narración y la psicología de los personajes. Rodríguez (1995) en su texto El oficio de escribir menciona al escritor Juarroz (1980) afirmando que: "la escritura nace del encuentro inexplicable e insólito de dos palabras que no parecían poder reunirse" " esa sorpresiva conjunción de las palabras" (p.17), "se entra en un plano de contemplación activa" (p.17), “ se va integrando algo así como un organismo verbal, cuyo desarrollo exige una entrega total, una plena disponibilidad y una fidelidad sin atenuantes" (p.17), " el punto de partida no es una simple improvisación, o un don divino, o una inspiración en el sentido más o menos mágico de la palabra... El punto de partida es un despertar" (p.17). 
En ese sentido Rodríguez (1995) menciona a Poe con la "filosofía de la composición; donde hay un propósito preliminar de construcción, y en donde los hallazgos son hijos de la previsión, el punto de partida para la escritura está ligado a un plan, al mapa, a una cierta carta de navegación inicial" (p.17).

En este sentido, el ejercicio de escritura requiere de tres hechos relevantes, que resultan en casi todos los casos desconocidos para los aprendices de la escritura, el primero es la lectura. Autores, pensadores y críticos literarios están a favor de que la buena lectura fortalecerá el proceso para quien quiera dedicarse a la escritura. Nos recuerda Díaz Robello (1980) en una entrevista cuando cita una frase célebre del escritor Carlos Fuentes "Tienes que amar la lectura para poder ser un buen escritor, porque escribir no empieza contigo" (p.5).

Aquí se presenta entonces un primer reto. La mayoría de los estudiantes de la universidad ingresan a las carreras de humanidades con intenciones artísticas y literarias, pero sin haber leído casi nada en toda su formación escolar y con deficiencias en comprensión lectora y redacción. Como lo afirma Cassany (1997) citado por Niño-Rojas (2011 p. 156) "escribir no es poner letras o signos en un papel en blanco, sino elaborar un significado global y preciso sobre un tema y hacerlo comprensible para la audiencia, utilizando el código escrito".

El segundo hecho es la disciplina que se podría explicar diciendo que es la práctica del oficio mismo. Escribir necesita tiempo y mucha práctica como lo necesitaría cualquier otra profesión u oficio. VásquezRodríguez (1995) recurre a García Márquez cuando habla de las puertas de acceso a la escritura: "una reconciliación con el tema a fuerza de tenacidad y dominio, otros apelarán a documentos, a la investigación detallada y rigurosa; algunos confiarán a la fuerza de un recuerdo, de un olor, o echarán mano de las huellas o improntas de la infancia" (p. 18).

La escritura es comparable con el deporte, se debe entrenar todos los días, no se corren cien metros planos si nunca se sale a ejercitar, entonces para lanzarse a la aventura de construir un texto se debe escribir constantemente, Mempo Giardinelli dice "calentar las ideas" (p.18). Sin la práctica necesaria, los textos escritos de vez en cuando, no serían suficientes para dominar el idioma y para acercarse a la creación literaria. La disciplina tarde o temprano logrará hacer que se domine la escritura.
Por último, el oficio versus la inspiración esta última se puede convertir en un mito a veces en un tropiezo. Inspiración vista como un acto divino solo para escogidos por la sagrada providencia para el arte de la escritura. Este concepto aleja al aprendiz de escritor del oficio, el trabajo diario, y los hace quedarse con el primer texto que escriben o con ese primer borrador. Vásquez-Rodríguez (1995) menciona a Nélida-Piñón quien hace hasta diez borradores de cada texto, "y no por obsesión formal, sino como un medio de liberar la escritura y alcanzar lo que tiene que ser dicho sin que nada lo ahogue y le impida asumir su verdadera naturaleza".

Acuño mi propia experiencia como docente señalando que en las aulas de clase es frecuente escuchar que: "No elaboré el escrito porque me faltaba inspiración" o "La inspiración no me llegó para escribir" Es indudable hay talentos innatos, plumas bendecidas, pero para el resto de los mortales la buena escritura se consigue trabajando a diario en el oficio de escribir. Orrantia (2012) en su artículo La escritura creativa en Colombia afirma: "Muchos académicos aún son escépticos al hablar de la profesionalización de la escritura, y no es para menos. No se atreven a decir que existe un auge, básicamente porque la historia de este país no es una historia donde la literatura haya sido fundamental para el desarrollo. "Este no fue un país de inmigrantes, un país donde hubiera vanguardia"(p.3)., dice Octavio Escobar, escritor y profesor de la Universidad de Manizales. Lo cierto es que esa endogamia y la satanización de las ideas nuevas convirtieron a la escritura en una actividad bohemia, romántica y poco importante. En este país se confía en los economistas, en los médicos, en los ingenieros, pero la escritura se ve como una profesión menor, y en algunos casos ni siquiera como un oficio, sino como un hobbie".

Es por ello, que el acercamiento a la escritura creativa se debe dar con la observación de su propia realidad como lo dice el autor White (1992). "Distingue entre un discurso histórico que narra y otro que narrativiza. El primero adopta abiertamente una perspectiva que observa al mundo y lo registra, mientras que el segundo es un discurso que simula hacer hablar al mundo mismo como un relato". Contursi y Ferro (2000, p.76.).

Los estudiantes y los docentes se niegan en muchas ocasiones a reflexionar sobre que el arte literario se pueda enseñar así lo confirma Orrantia (2012) "Aún hay muchos escritores que se niegan 
a participar en talleres, bien sea como profesores o como alumnos, porque creen que este es un talento y el talento no se enseña. Podría pensarse que tienen razón, pero cabe destacar que no es solo talento lo que se requiere para escribir. Ben Okri, escritor nigeriano ganador del premio Booker en el Reino Unido por su novela El camino hambriento, se aproxima a ese problema al señalar que: "Existe el arte y existe el artificio. El arte no se puede enseñar, el artificio sí. Se puede enseñar a escribir correctamente, pero no se puede enseñar que el escritor pueda sentir el alma de su tiempo. Eso es algo con lo que se nace. Hay momentos, sin embargo, en los que el artificio puede opacar el arte y el escritor talentoso sucumbe a la técnica y deja el arte a un lado" (p.3).

En este sentido encontramos deficiencias de los estudiantes en las competencias comunicativas básicas, no leen porque no tienen el gusto y hábito de hacerlo, tampoco escriben porque no saben cómo hacerlo, en este último existen problemas de redacción, ortografía, claridad de ideas y creatividad a la hora de abordar un tema.

Por lo tanto, los estudiantes de Comunicación Social de primer semestre vienen con deficiencias de producción lecto-escritora, que han tenido poco contacto con la literatura, pero que quieren aprender a escribir, tomaran los ejercicios propuestos de lectura y escritura para desarrollar la habilidad de escribir textos narrativos subsanando así las deficiencias lecto-escritoras.

Aprendizaje significativo citado por Moreira (1997) "es el proceso a través del cual una nueva información (un nuevo conocimiento) se relaciona de manera no arbitraria y sustantiva (no-literal) con la estructura cognitiva de la persona que aprende. En el curso del aprendizaje significativo, el significado lógico del material de aprendizaje se transforma en significado psicológico para el sujeto". Moreira (1997) que cita a Ausubel (1963, p. 58), explica que: "el aprendizaje significativo es el mecanismo humano, por excelencia, para adquirir y almacenar la inmensa cantidad de ideas e informaciones representadas en cualquier campo de conocimiento".

La influencia del aprendizaje significativo de Ausubel (1963) en el desarrollo de las técnicas de escritura creativa de Rodari (1999), responde a la necesidad encontrada por los docentes de redacción en el programa de Comunicación Social de la Universidad de Pamplona, donde preocupan las pocas habilidades lecto-escritoras que tienen los estudiantes de primer semestre, los bajos niveles de lectura, la falta de coherencia en los escritos, la apatía frente a la escritura sea ficción o no ficción. Guillen 2020 "Por lo tanto, el uso de la imagen como medio para la enseñanza-aprendizaje requiere darse bajo estrategias pedagógicas apropiadas en el aula; permitiendo la construcción colectiva e interactiva de los saberes". En este caso hablamos de la imagen como detonador o disparador para la creación literaria.

Esta investigación pretende corregir esas debilidades de escritura, generar hábitos lectoescritores, despertar el interés en la escritura literaria por medio de las técnicas de Rodari (1999) y las prácticas pedagógicas de la teoría del aprendizaje significativo, teoría que introdujo Ausbel en 1963. Esta teoría es relevante en el desarrollo de este proyecto ya que se tomará el aprendizaje significativo de proposiciones por combinación, que abarca esquemas con diferentes tipos de conocimiento sobre la realidad, como son: hechos, acontecimientos, experiencias, anécdotas personales, miradas de la realidad pueden llegar a ser fundamentales a la hora de desarrollar procesos de escritura creativa. El porqué de este estudio. Asimismo, la investigación se justifica desde tres perspectivas social, metodológica y teórica.

En este sentido desde lo social por que el método vivencial de escritura creativa se apoya en el aprendizaje significativo para lograr motivar y desarrollar la escritura con los estudiantes. Se dará en dos momentos en el proceso metodológico: El primero la motivación, "La lectura es un instrumento potentísimo de aprendizaje: leyendo libros, periódicos o papeles podemos aprender cualquiera de las disciplinas del ser humano" (p.193). Cassany, Luna, Sanz. (1997, p.193). Desde la lectura que generará avances de los estudiantes en comprensión lectora a través del aprendizaje de las dimensiones, los factores y las operaciones de la lectura. Este primer momento en el proceso de escritura es fundamental ya que debe despertarse la sensibilidad, la imaginación, la creatividad y la motivación por el uso creativo de la palabra. "En la lectura hay que imaginar... la lectura es un acto de creación permanente" Cassany. (1995, p. 193).

En un segundo momento se propiciará la escritura a través de ejercicios de lectura del contexto, de la realidad social, llamado escritura creativa vivencial. Para esto se utilizaron detonadores o disparadores de la escritura, o juegos que promueven 
la escritura en el aula. Cáñaz (2015 p. 22) afirma en este sentido "Se reconoce que la lectura y la escritura son procesos complejos que implican en los niños capacidades cognitivas desarrolladas al máximo para que puedan concentrarse; en este proceso el juego les ayuda a que la actividad mental sea constante, lo cual contribuye al desarrollo del cerebro".

Esto a través de las técnicas creativas propuestas por el pedagogo y escritor Rodari. El autor plantea la importancia de cultivar la fantasía en los niños, en los jóvenes, y plantea una postura crítica sobre los ambientes escolares una de sus afirmaciones las plantea en el texto Gramática de la fantasía donde afirma: “¿Merece la pena que un niño aprenda llorando lo que puede aprender riendo?" Rodari (2002 p. 16).

Finalmente, desde la perspectiva teórica, ya que se van a fortalecer conceptos, y teorías sobre la enseñanza - aprendizaje, de la creación literaria. La investigación, la buena escucha y la reflexión son habilidades que se propiciarán para que el estudiante pueda generar un proceso de escritura significativo. La importancia del desarrollo del proyecto radica en la experiencia que adquirirá el partícipe en el estudio del lenguaje y la palabra a través de los procesos de lecto-escritores. Cómo dejan una memoria de su propio contexto por medio de las historias elaboradas.

\section{Diseño de la Investigación}

El diseño que se llevó a cabo para el desarrollo del proyecto es el fenomenológico, Taylor y Bogdan (1984), lo definen como el estudio de los fenómenos sociales desde la perspectiva de los actores sociales "Dentro de esta corriente, destacan Taylor y Bogdan (1984), cuya perspectiva se basa en una fenomenología esencial: "Para el fenomenólogo, la conducta humana, lo que la gente dice y hace, es producto del modo en que define su mundo. La tarea del fenomenólogo y de nosotros, estudiosos de la metodología cualitativa, es apre-hender este proceso de interpretación. Como lo hemos subrayado, el fenomenólogo intenta ver las cosas desde el punto de vista de otras personas." (p.9).

La fenomenología se fundamenta en las siguientes premisas (Hernández, Fernández, \& Baptista, 2006, p. 712-713):

- "En el estudio, se pretende describir y y entender los fenómenos desde el punto de vista de cada participante y desde la perspectiva construida colectivamente".

- "El diseño fenomenológico se basa en el análisis de discursos y temas específicos, así como en la búsqueda de sus posibles significados".

- "El investigador contextualiza las experiencias en términos de su temporalidad (tiempo en que sucedieron), espacio (lugar en el cual ocurrieron), corporalidad (las personas físicas que la vieron) y el contexto relacional (los lazos que se generaron durante las experiencias)".

Por otra parte, el estudio de la escritura creativa que implica conceptos como la imaginación, creatividad y la producción textual; encuentra en el modelo de fenomenología una contribución a su estudio enmarcado en una visión para comprender los conocimientos, fuentes del pensamiento creativo. En este proyecto de investigación se le da especial énfasis a la experimentación y la escritura vivencial como parte del aporte del modelo de aprendizaje significativo y por lo tanto el diseño fenomenológico permite analizar las fases de aprendizaje dentro de la aplicación de las técnicas de Rodari quien hacia énfasis en los ambientes escolares y las vivencias como parte de la formación del conocimiento.

La presente investigación se enmarca dentro de la investigación cualitativa ya que se va a trabajar desde la experiencia de los estudiantes con el aprendizaje de la escritura literaria. Dado que:

\footnotetext{
Por medio de un conjunto de técnicas o métodos como las entrevistas, las historias de vida, el estudio de caso o el análisis documental, el investigador puede fundir sus observaciones con las observaciones aportadas por los otros" Rodríguez Gómez Gregorio, Gil Flores Javier, García Jiménez Eduardo (1996, p.62).
}

Por otra parte: "Lo que distingue a este diseño de otros diseños cualitativos es la o las experiencias del participante o participantes como centro de indagación (Hernández, Fernández, \& Baptista, (2006, p. 712). La base de la fenomenología es que existen diversas formas de interpretar la misma experiencia, y que el significado de la experiencia para cada participante es lo que constituye la realidad Hernández, Fernández, \& Baptista, (2006, p. 712)”. 


\section{Resultados}

En la teoría de Ausbel (1963) el rol del docente en el aprendizaje significativo es de guía o adopta una posición de líder para llevar a que los estudiantes participen de un proceso de aprendizaje que implica una introspección de su ser para generar las historias. La creación de historias o la producción literaria si se quiere llamar así, no es un proceso azaroso o fortuito; por el contrario, requiere una constante reflexión; lectura del mundo no solo en el instante en que se está creando la historia si no debe ser un trabajo diario, constante que despierte en el aprendiz de escritor una sensibilidad especial para leer el mundo, interpretarlo y construir una nueva realidad fantástica. Rodari (1999) cita al libro "Fragmentos de Novalis (17721801): "Si tuviéramos también una Fantástica, como una Lógica, habríamos descubierto el arte de inventar" (p.13).

En este sentido deberíamos generar un proceso de aprendizaje, desarrollar la "fantástica" como la llamaría Novalis (1978), como lo refiere Rodari (1999); quién además reflexiona sobre lo siguiente: "Porque siempre hay un niño que pregunta: ¿Cómo se hace para inventar historias? y merece una respuesta honesta" (p.15).

Esa respuesta se encuentra a través de experimentación, el desarrollo de talleres generando vivencias significativas de aprendizaje con inclusión de los detonadores o disparadores de escritura, que a su vez generan la apertura a la imaginación, la fantasía, pero también al proceso, la disciplina y la retroalimentación del ejercicio creativo.

\section{Conclusiones}

En este proyecto de investigación se estudió la influencia del aprendizaje significativo de Ausbel (1963) en el desarrollo de las técnicas de escritura creativa de Rodari (1999) a través del desarrollo del proyecto de investigación se pudo llegar a las siguientes conclusiones:

El descubrir en un proceso de enseñanza de creación literaria a través de un estudio del contexto, el ambiente escolar; las habilidades de los estudiantes ya sea para descubrirla o para potenciarla; contribuye a los procesos educativos por un lado los participantes mejoran elementos cognitivos como la memoria, la resolución de conflictos, lógica, imaginación; aunque no sea un objetivo central también tendrán una mejor ortografía y gramática.
Los estudiantes lograron apropiarse de términos antes desconocidos como los son los detonadores, la verosimilitud y la fantástica. En este sentido se logró realizar un proceso de enseñanza aprendizaje en torno a potenciar el desarrollo de la fantástica como habilidad cognitiva.

Utilizando la técnica correcta en el ambiente y con el estímulo adecuado se puede generar producción textual literaria en los estudiantes. Se concluye a través de este trabajo de investigación que es posible generar un proceso creativo en cualquier persona utilizando las herramientas pedagógicas y los estímulos adecuados. En este sentido con este proyecto se desmitificó la escritura por inspiración y se fortalece la idea que la escritura requiere un trabajo, conocimientos previos y aplicación de técnicas que faciliten la imaginación y la creación literaria.

\section{Referencias}

Ausubel (1963). The Psychology of Meaningful Verbal Learning. Congreso Phi, Delta, Kappa.

Cáñaz (2015). Ensayo académico. Importancia del juego en el proceso de lecto escritura de niños de 6 a 7 años. Recuperado

de: http://dspace.uhemisferios.edu.ec:8080/xmlui/bitstream/ handle/123456789/209/tesis\%20final\%20corregida.pdf ?sequence $=1 \&$ isAllowed $=\mathrm{y}$

Cassany. D. (1997). Describir el escribir. Cómo se aprende a escribir. Anagrama.

Contursi, M. E. \& Ferro, F. (2000). La narración usos y teorías. p. 76.

De Dios-Menéndez, V. (1980) Entrevista a Eva Díaz Riobello Microtextualidades. Revista Internacional de microrrelato y minificción. N. 2, pp. 132137.

Domínguez Y.\& J. Guillermo (2002). ¿Por qué no escriben textos los estudiantes? (Parte1). Revista del Centro de Investigación. Universidad La Salle, 5 (19), 85-98. ISSN: 1405-6690. Recuperado de: https://www.redalyc.org/articulo.oa?id=342/34251911

Guillen, G. (2020). La pedagogía de la imagen como forma de promover el aprendizaje significativo dentro del aula. Revista Tecnológica-Educativa Docentes 2.0, 9(1), 96-108. https://doi.org/10.37843/rted.v9i1.90

Hernández-Sampieri, R., Fernández-Collado, C., \& Baptista-Lucio, P. (2006). Metodología de la investigación (4th ed.). McGrawHill. 
Moreira, M. (1997). Aprendizaje significativo: un concepto subyacente. Instituto de Física Porto Alegre, RS, Brasil 1997.

Niño (2011). Competencias en la comunicación. Hacia las prácticas del discurso. Ecoe Ediciones.

Novalis (1978). Fragmentos y anotaciones sobre poesía y filosofía traducción de Nicolás Trujillo Osorio. Cuadro de tiza ediciones-Chile.

Orrantia, M. (2012). La escritura creativa en Colombia. Literatura: Teoría, Historia, Crítica. 14 (1), 287-301. Recuperado de: http://www.scielo.org.co/scielo.php?script=sci_arttext\&pid=S 0123-59312012000100014\&lng=en\&tlng=es.

Penacc. D. (2006). Como una novela. Grupo Editorial Norma.

Rodary. G. (1999). Gramática de la fantasía. Introducción al arte de contar historias. Panamericana.

Rodríguez-Gómez, G., Gil-Flores, J., García-Jiménez, E. (1996). Metodología de la investigación cualitativa. Aljibe.

Taylor \& Bogdan (1984). Introducción a los métodos cualitativos de investigación. Paidós.

Vásquez-Rodríguez, F. (1995). Signo y Pensamiento. El oficio de escribir. PP15-24 Recuperado de: https://www.javeriana.edu.co/signoyp/pdf/2602.pdf 